\title{
RANS and LES Modeling of the GE10 Burner
}

\author{
Vladimir L. Zimont ${ }^{1}$, Vincent Moreu ${ }^{1}$, Valerio Battaglia ${ }^{1}$, Roberto Modi $^{2}$ \\ ${ }^{1}$ CRS4, Science and Technology Park Polaris, Pula, Italy \\ ${ }^{2}$ GE Oil \& Gas Nuovo Pignone, Florence, Italy \\ E-mail: \{zimont,moreau\}@crs4.it, roberto.modi@ge.com \\ Recieved April 28, 2011; revised May 29, 2011; accepted June 10, 2011
}

\begin{abstract}
The paper presents 1) the numerical results of RANS (Reynolds Averaging Navier-Stokes) simulations for two versions of the premixed combustion GE10 burners: the old one with non-premixed and modified one with swirled premixed pilot flames; and 2) the numerical results of joint RANS/LES (Large Eddy Simulation) modelling of the ONERA model burner and a simplified GE10 combustor. The original joint RANS/LES approach is based on using the Kolmogorov theory for modelling sub-grid turbulence and combustion intensity and using RANS numerical results for closure the LES model equations. The main conclusion is that developed joint RANS/LES approch is the efficient timesaving tool for simulations both the average and instantaneous fields of parameters in gas turbine and boiler burners with premixed combustion.
\end{abstract}

Keywords: Turbulent Premixed Combustion, Gas Turbine Burner, Joint RANS/LES Simulation

\section{Introduction}

The proposed work is devoted to lean premixed combustion technology, which is nowadays well established within industrial gas turbine industry in order to reduce nitric oxides $\left(\mathrm{NO}_{\mathrm{x}}\right)$ emission. The main numerical modeling tool for industrial gas turbine combustion is RANS codes, which yield averaged fields and integral characteristics of the flow. The main part of this presentation is devoted to RANS simulations of two variants of the GE10 gas turbine combustor, which include 1) simulations of the premixed combustion in the chamber; 2) simulations of the preliminary partial mixing of gas fuel and air; 3) simulations of the non-premixed pilot flame (old version GE10); 4) premixed pilot flame (new version GE10); and 5) air jets cooling system of the chamber. In conditions of industrial gas turbines, instanttaneous combustion takes place in non-laminar (microturbulent) and strongly wrinkled sheets with small-scale structure, which fundamentally cannot be resolved by model RANS and LES equations. In the presented simulations, we used our Turbulent Flame Closure (TFC) model [1-3], where this fundamental problem of modeling ("challenge of turbulent combustion") is resolved in the context of the Kolmogorov hypothesis of statistical equilibrium of small-scale turbulent structures generalized for the case of turbulent combustion. This model was already used for RANS simulations of the gas tur- bine combustion and these results where presented in IGTACE, Florida, 1997 (97-GT-395) published in [4] and JPGC, 2001 [5]. (This model is now implemented in the commercial codes Fluent and CFX.)

RANS results are important but not sufficient as nonstationary characteristics of the flow are also important in gas turbine applications. In academicals works, an attempt to replace RANS tool by LES one is ongoing. We think that "LES instead of RANS" in industrial application is untimely and we proposed in [6] a joint RANS/LES approach where the mean fields are simulated by the RANS tool while the corresponding nonstationary fields are simulated by the LES one using for modeling some information from the preceding RANS simulation. The paper [6] contains numerical illustrations of this approach concerning mainly "academic" flames. We achieved agreement between RANS and LES subproblems by using in fact the same combustion models in both sub-problems. In this paper, we present numerical results in the context of the joint RANS/LES approach for gas turbine combustors.

We found support to our approach in the invited lecture [7]. In the conclusions, the authors write: "The future tools for gas turbine designs will be based on classical Reynolds Averages codes to predict main flows but will also rely on Large Eddy Simulation tools coupled to acoustic codes" (in our example both RANS and LES sub-problems were stated in incompressible formulation). 
We notice that proposed in this paper approach was discussed at the ASME ATI Conference in Italy "Energy: production, distribution and conservation” in May, 2006 and was submitted to the proceeding of the conference, but it was not published in a journal. We use this joint RANS/LES approach in our work with the industries and hope that this publication would be useful for engineers working in the field modeling of industrial combustion.

\section{Principles of Modelling and the Equations}

In this section we briefly describe a physical model of premixed combustion at strong turbulence and fast chemistry and present the equations used for both RANS and LES simulations.

\subsection{The Physical Model}

In gas turbines, typical turbulent Reynolds numbers $\operatorname{Re}_{t}=u^{\prime} L / v$ are large and typical Damkohler numbers $D a=\tau_{t} / \tau_{c h}$ are moderately large. Here, $\tau_{t}=L / u^{\prime}$ and $\tau_{c h}=\chi / S_{L}^{2}$ are the turbulent and chemical times, $\chi$ is the molecular heat conductivity and $S_{L}$ is the speed of the normal laminar flame, these parameters being used in the model as the physico-chemical characteristics of the reactant. In this case, the speed $U_{f}$ and width $\delta_{f}$ of the thickened flamelets are controlled by the statistically equilibrium small-scale turbulence and the chemical time $\tau_{t}$, and it results the following expressions [1-3]:

$$
U_{f} \approx u^{\prime} \cdot D a^{-1 / 2}>S_{L}, \delta_{f} \approx L \cdot D a^{-3 / 2}>\delta_{L} .
$$

The flamelet broadening takes place at $\delta_{L}>\eta=L \mathrm{Re}_{t}^{-4 / 3}$, i.e. when the size of the minimal eddies $\eta$ is less than the laminar flame width $\delta_{L}$. At the same time, the thickened flamelet sheet is strongly wrinkled by turbulence when $L \gg \delta_{f}$ and hence the condition for this combustion mechanism is the following [1-3]:

$$
D a^{3 / 2} \gg 1 \gg D a^{3 / 2} \operatorname{Re}_{t}^{-4 / 3}
$$

So the model is valid at $\operatorname{Re}_{t} \sim\left(10^{2}-10^{3}\right)$ and $D a \sim 10^{1}$; these conditions are common for large-scale gas turbine combustors. For weaker turbulence, common for smallscale laboratory burners, instantaneous turbulent combustion takes place in a wrinkled laminar flamelet sheet. Real combustion takes place in transient flames with increasing width. The reason is that the large-scale wrinkles of the flamelet sheet remain statistically in non-equilibrium at real residence time in the combustor. So the width is controlled by the turbulent diffusion coefficient $D_{t}$ quite similarly to the nonreacting mixing layer. At the same time, the small-scale winkles can be assumed statistically in equilibrium and as they give the main contribution in the dimensionless flamelet sheet area
$\left(\bar{F} / F_{0}\right)$, the turbulent flame velocity $U_{t}=U_{f}\left(\bar{F} / F_{0}\right)$ is controlled by large-scale turbulence parameters (in the model, they are the r.m.s. velocity pulsation $u^{\prime}$ and the integral scale $L$ ) and the chemical time $\tau_{t}: U_{t} \approx u^{\prime} D a^{1 / 4}[1-3]$.

\subsection{RANS Equations of the TFC Premixed Combustion Model}

We analyze premixed flames adopting the flamelet formalism in terms of the progress variable c, using the known bimodal approximation of the PDF $\mathrm{p}(\mathrm{c})$ where the width of the thin instantaneous reaction zone is ignored. In this case, we have the following expressions:

$$
\begin{aligned}
& \bar{\rho} / \rho_{u}=\left(1+\tilde{c}\left(\rho_{u} / \rho_{b}-1\right)\right)^{-1}, \\
& \tilde{T}=T_{u}(1-\tilde{c})+T_{b} \tilde{c}, \\
& \tilde{C}_{i}=C_{i, u}(1-\tilde{c})+C_{i, b} \tilde{C},
\end{aligned}
$$

where $\rho_{u}, T_{u}, C_{i, u}$ and $\rho_{b}, T_{b}, C_{i, b}$ are the density, temperature and species concentrations in unburned (reactants $\tilde{c}=0$ ) and burned (equilibrium products $\tilde{c}=1$ ) gases.

The turbulent combustion front, moving with speed $U_{t}$ and having increasing brush width controlled by $D_{t}$, is described by the following transport equations:

$$
\begin{aligned}
& \partial \bar{\rho} \tilde{c} / \partial t+\nabla \cdot(\bar{\rho} \tilde{\boldsymbol{u}} \tilde{c}) \\
& =\nabla \cdot\left(\bar{\rho} D_{t} \nabla \tilde{c}\right)+\rho_{u} U_{t}|\nabla \tilde{c}|(a), \\
& U_{t}=\mathrm{A}^{3 / 3} S_{L}^{1 / 2} \chi_{u}^{-1 / 2} L^{1 / 4}(b),
\end{aligned}
$$

where $\mathrm{A}$ is an empirical constant equal to $\mathrm{A} \approx 0.5$ valid for all fuels tested in [8,9] (methane $\mathrm{CH}_{4}$, ethane $\mathrm{C}_{2} \mathrm{H}_{6}$, propane $\mathrm{C}_{3} \mathrm{H}_{8}$ and even for hydrogen $\mathrm{H}_{2}$ ). Equations (4) are combined with the average hydrodynamic equations and the " $k-\varepsilon$ ” model.

\subsection{The LES Equations of the TFC Model}

The main idea of our joint RANS/LES approach [6] is to combine LES and RANS in a two-stage process. The first step consists of the RANS simulation which yields the averaged flow field; the second step entails LES using the mean turbulent dissipation rate $\varepsilon(\vec{x}, t)$ obtained from RANS to estimate the subgrid turbulence $u_{\Delta}^{\prime}$ and subgrid flame speed $U_{t}^{\Delta}=U_{f}\left(\bar{F}_{\Delta} / F_{0}\right)$. LES gives nonstationary images of a RANS simulation.

The model LES equation can be cast as follows:

$$
\begin{aligned}
& \partial\left(\bar{\rho}_{\Delta} \tilde{c}_{\Delta}\right) / \partial t+\nabla \cdot\left(\bar{\rho}_{\Delta} \tilde{u}_{\Delta} \tilde{c}_{\Delta}\right) \\
& =\nabla \cdot\left(\bar{\rho} D_{\Delta} \nabla \tilde{c}_{\Delta}\right)+\rho_{u} U_{t}^{\Delta}|\nabla \tilde{c}|
\end{aligned}
$$


where the subgrid flame speed and subgrid transport coefficients are the following:

$$
\begin{aligned}
& U_{t}^{\Delta}=A^{*} u_{\Delta}^{\prime} S_{L}^{1 / 2} \chi_{u}^{-1 / 4} \Delta^{1 / 4}\left(\approx U_{t}(\Delta / L)^{1 / 2}\right)(a), \\
& u_{\Delta}^{\prime} \approx \varepsilon^{1 / 3} \Delta^{1 / 3}(b), D_{\Delta} \approx v_{\Delta} \approx \varepsilon^{1 / 3} \Delta^{4 / 3}(c) .
\end{aligned}
$$

Use of the Kolmogorov viscosity $v_{\Delta}$, which in contrast to the commonly employed Smagorinsky model of sub-grid turbulence does not depend on time, makes LES modeling more numerically favourable.

In our analysis, we paid special attention to the problem of consistency of the results of RANS and average LES sub-problems, as it is a key point of the consistency of joint RANS/LES approach. This correspondence is based on using the same combustion model in both sub-problems. A difference in the hydrodynamic pictures can be in the situation where " $k-\varepsilon$ " turbulence model is not accurate. In the presented simulations it is not this case.

\section{Burner Descriptions and Simulations}

We present the result of RANS numerical simulations of two versions on the NP GE10 gas turbine combustion system, which use non-premixed and premixed pilot flames. The examples presented not only refer to the simulation of the combustor aerodynamics: the main and pilot turbulent flames, cooling air jets and so on, but also to the mixing process in the premixing channel and the flow inside the premixed pilot burner. All the simulations presented in this section have been performed with StarCD (V.3.15) complemented with user subroutines. Reynolds numbers are in the range $10^{6}-10^{7}$ and the turbulent flow has been simulated using the “ $k-\varepsilon$ ” turbu- lence model with wall functions.

\subsection{Premixing Channel}

This part is comprehensive of all the mixing channel up to the combustion chamber inlet and goes upstream quite before the system which controls the mass flow rate, as shown in left. Taking into account the symmetries of the problem, we have restricted the simulation to an angular sector of 30 degrees. Inside this sector, there are two half-winglet (lying on the cyclic boundaries), two fuel nozzles and one opening of the flow controller. We used about 2 millions cells which was the maximum reasonably acceptable for stationary computations with the computational power available.

When the flow controller is not fully open, it induces some swirl component in the flow. For the open configuration, the symmetry is higher and we could have expected the flow to have a symmetrical pattern of 15 degrees opening. Nevertheless, as shown on Figures 1(b)(d), the numerical solution is very far away from the symmetrical expectation, at least concerning the flow investing the fuel nozzles. The winglet downstream has the effect to strongly damp an eventual global swirl component, but a departure of moderate strength from the uniform axial velocity profile at the chamber entrance. Moreover, the fuel concentration also showed a not so moderate dispersion, warning about an eventual non-perfect mixing of the instantaneous fuel concentration. It is not yet known whether the discrepancy from a symmetrical solution comes from numerical limitation or is an indication that the symmetrical solution is really unstable.

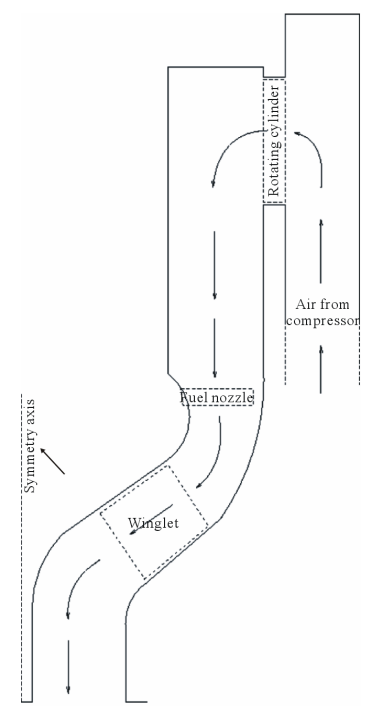

(a)

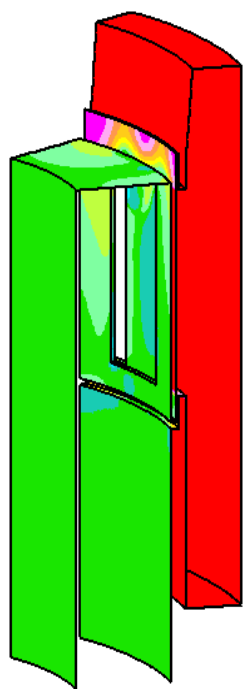

(b)

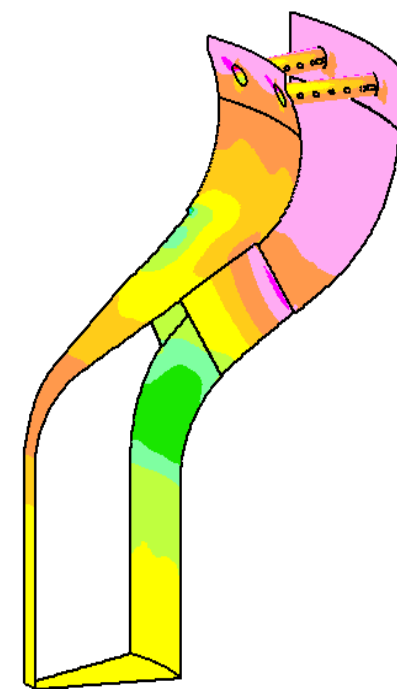

(c)

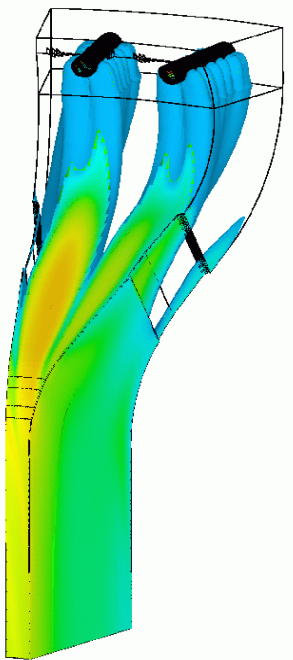

(d)

Figure 1. (a): the mixing channel; (b) and (c): the pressure colored wall boundaries; (d) mixing coloured by the fuel concentration. 


\subsection{GE10 Burner with Non-Premixed Pilot Flame}

One forth of the combustion chamber has been simulated, Figure 2. It includes 6 pilot burners at the chamber entrance and a variety of small secondary cold air inlet disposed in rows and aimed at cooling the chamber wall. It also includes one dilution air inlet close to the chamber exit. The smallest inlet hole rows have been collectively simulated as inlet annuli with the same mass flow rate. The main flow inlet condition has been taken from the mixing channel simulation with a cyclic replication to obtain a 90 degree sector. The flame development is very close to axial-symmetry. The small departures caused by the main inlet condition and by the pilot burners do not have large scale consequences. Intensive combustion takes place in the relatively small part of the chamber. Figure $\mathbf{3}$ shows the mesh and 3D field of the temperature.

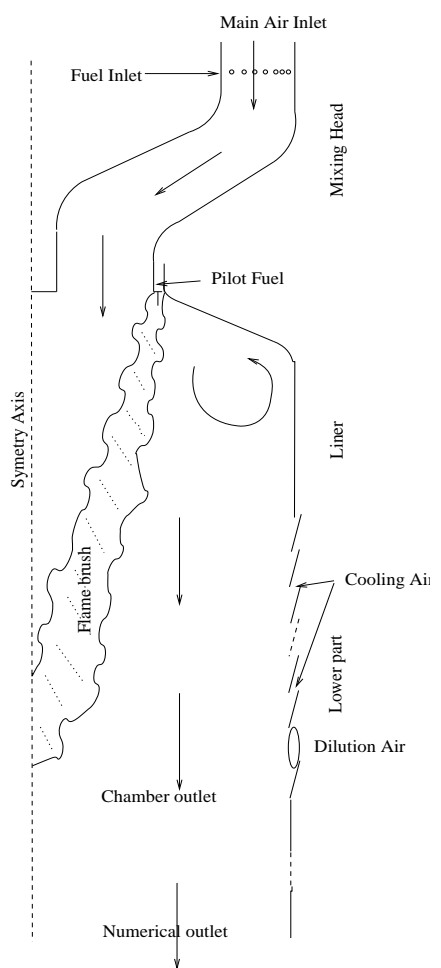

(a)

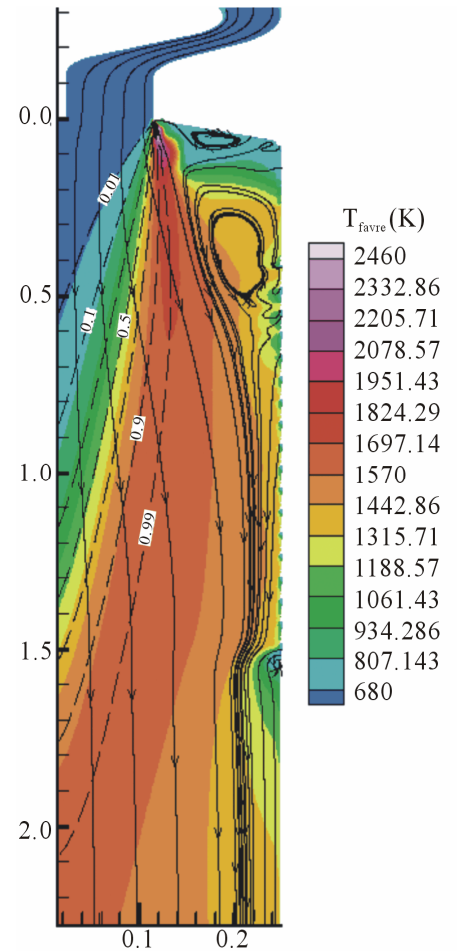

(b)

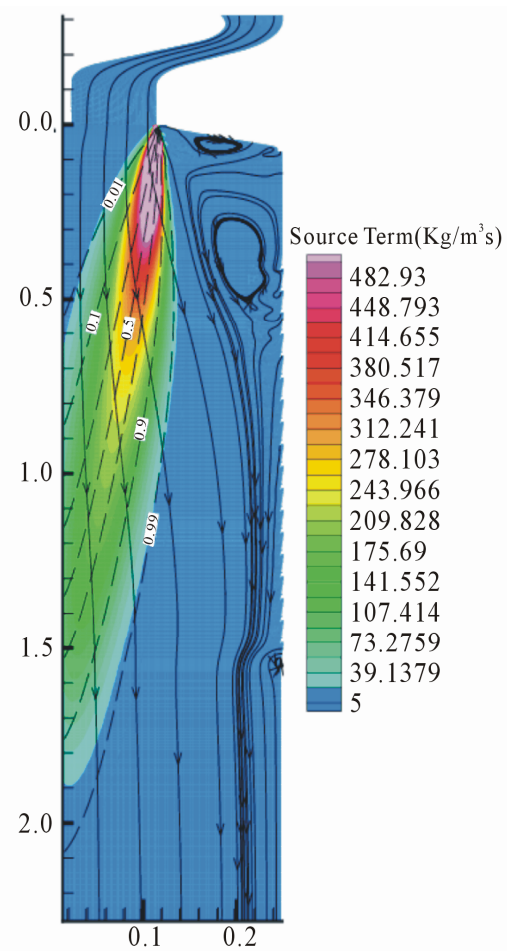

(c)

Figure 2. (a): Sketch of the combustion chamber; (b): RANS simulations of the combustor GE10 colored by the temperature; (c): the model source term with the flow streamlines and iso-contours of the progress variable.

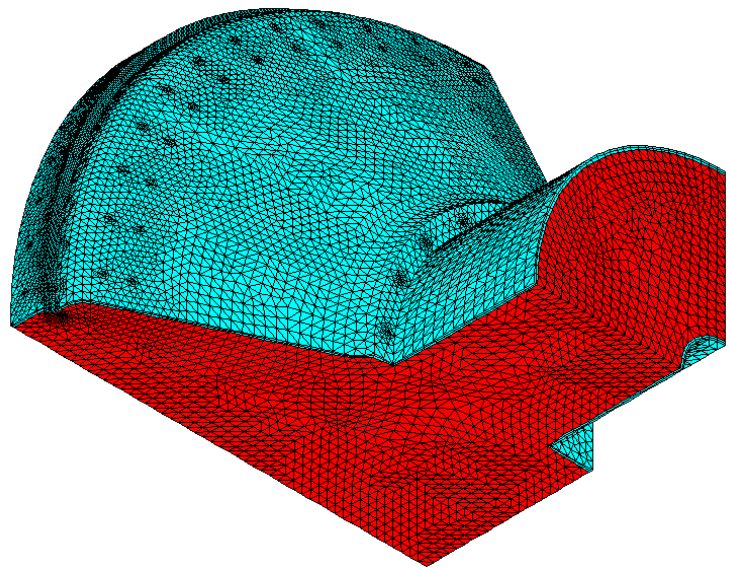

(a)

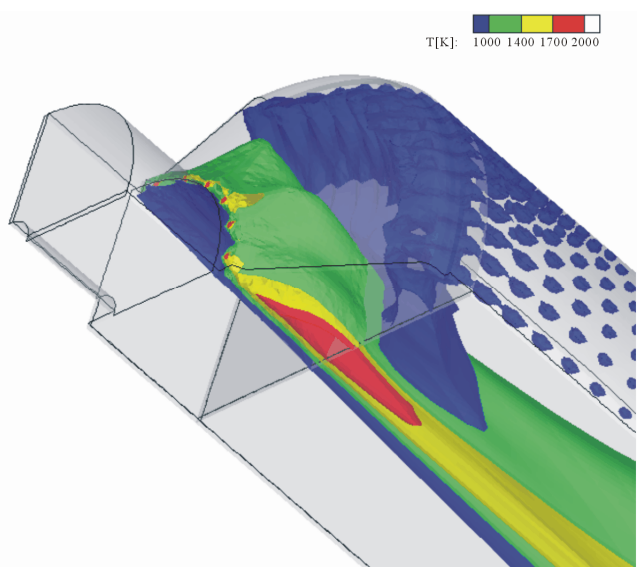

(b)

Figure 3. (a): Mesh of the combustion chamber inlet and cap; (b): 3D isosurfaces of the temperature 1000-1400-1700-2000K. 


\subsection{GE10 Combustor with Premixed Pilot Flame}

To lower to $\mathrm{NO}_{\mathrm{x}}$ formation, GE decided to test a variant of the combustor where the 24 non-premixed pilot burners were replaced by 4 bigger but essentially premixed pilot burners. This procedure allowed having less fuel burnt in stochiometric condition. The pilot burner has been numerically tested in stand-alone condition. Figure 4, left, shows the fuel concentration that remains high only on a very narrow central region in exit of the burner. In Figure 4, centre, we see how the flame develops on an axial plane. Figure 4, right, show the new pilot burner as been numerically tested inserted in the combustion chamber cap. The global reacting flow field is somehow disturbed by the presence of the new pilot, Figure 5, and, obviously, is not any more close to axial-symmetrical. Mainly, the recirculation region is heavily perturbed as it is shown on Figure 6, left and centre. The interaction of the hotter pilot flame with the colder main flame can be appreciated in Figure 6, right.

\section{Joint RANS/LES Modelling of Turbulent Premixed Gas Turbine Combustion}

To understand the non-stationary features of the flow, we performed a LES analysis of the burner. We used our Joint RANS/LES approach [6] that strongly shortens the LES analysis. The simulations were performed using the Fluent package, a finite volume code which gives the possibility to customize the models implemented. It has a second order centered scheme fit to LES simulations which require low dissipative numerical schemes.

In the next paragraphs, we present the validation of this approach for a standard test case and then we show results of the LES for a simplified geometry of the GE burner previously described, not considering pilot system and cooling jets.

\subsection{The ONERA Standard Burner}

To validate the Joint RANS/LES approach, we used a standard test case known as the Moreau (ONERA) burner [10]. It consists in a rectangular section burner with the flame stabilized by a burned gas flow. The fuel is a methane-air mixture with equivalence ratio equal to 0.84 . The flow structures is mainly $2 \mathrm{D}$ due to the high aspect ratio of the cross section, so it is possible to perform 2D simulations without losing accuracy in the results. In this test case, LES simulations are very sensitive to inlet boundaries conditions for turbulence; in the present work, we introduced a disturbance in the average inlet velocity derived by the amplitude and length of the upstream flow turbulent characteristics reported in [10].

The results of both RANS and LES approaches, Figure 7 , show that the turbulent premixed flame has increasing brush width and at the same time nearly constant speed, as it can be seen by the practically constant angle with respect to the main flow. In the figure measured in the experiment mean temperature $\mathrm{T}$ and velocity $\mathrm{U}$, and the turbulent parameters (the rms fluctuation of the velocity u' and the integral scale L) have indexes "u" and "b" that refer correspondingly to the flows of the unburned and burned gases at the entrance of the burner. The upper graph represents a result of the RANS simulation of the progress variable $\tilde{c}$ : five isolines and two profiles in the sections $x=0.1$ and $x=0.5$. The lower graph represent the LES results: five isolines of the instantaneous progress variable and following from averaging of the LES result mean profiles $\tilde{c}$ for $\mathrm{x}=0.1$ and $\mathrm{x}=0.5$ and isolines of the mean progress variables.

The isolines, which directly follow from LES modeling, clearly show the instantaneous structure of the flame with the effects of the large scale vortices that convoluted
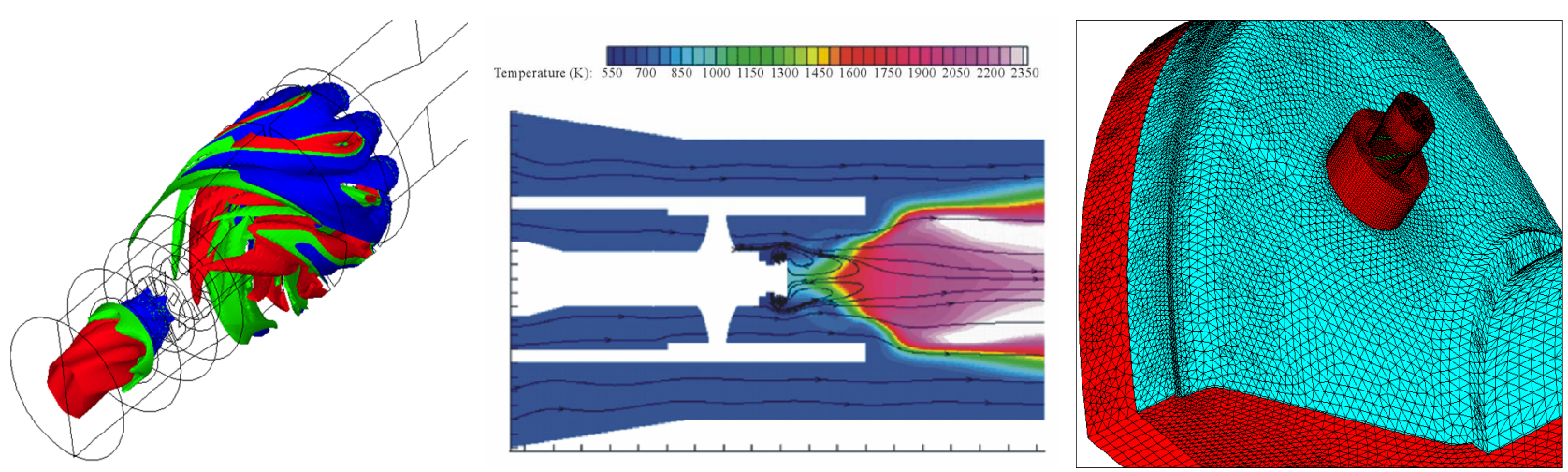

Figure 4. Left: premixed pilot burner with 2 coaxial swirling flows, coloured by the fuel concentration, the external flow arrives already mixed in the burning region and only the central part keeps a high fuel concentration; center: pilot burner flame with streamlines coloured by temperature; right: the computational mesh of the combustion chamber cap with the pilot burner inserted. 


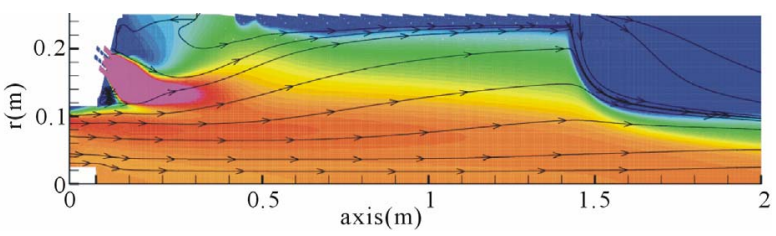

Figure 5. Field of equivalence ratio $\Phi$ with stream lines in a section of the burner.

and stretched the isosurfaces inside the flame. Comparison of the mean isolines and the profiles of the progress variables simulated directly by the RANS simulation and by averaging of the LES data (the upper and lower graphs in Figure 7) are similar. Figure 8 shows that the mean profiles of the progress variable and the velocity, which follow from the RANS and LES approaches, are close and they are in reasonable agreement with the experimental data from [10]. Measurements of r.m.s. velocity or tem- perature are not available to verify the accuracy of the fluctuations resulting from the simulations, but observed agreement between RANS and average LES data is a clue of the reliability of the instantaneous data.

The lower graph in Figure 7 represent also a linear distribution of a passive concentration $z$ in the entrance section $\mathrm{x}=0$, which was used in the simulations as a boundary condition, and following from averaging of the LES results the mean profiles $\tilde{z}$ in the section $\mathrm{x}=0.1$ and $\mathrm{x}=0.5$. Performed in [6] comparative analysis of the LES data for the progress variable $c$ and the passive concentrations $z$ showed that in the flame the mean flux of the passive concentration $z$ is gradient, while the mean flux of the progress variable $c$ is predominantly countergradient: it is gradient in the beginning of the flame and then becomes counter-gradient in the main part of the flame. The reason is that the flux of the passive concen-
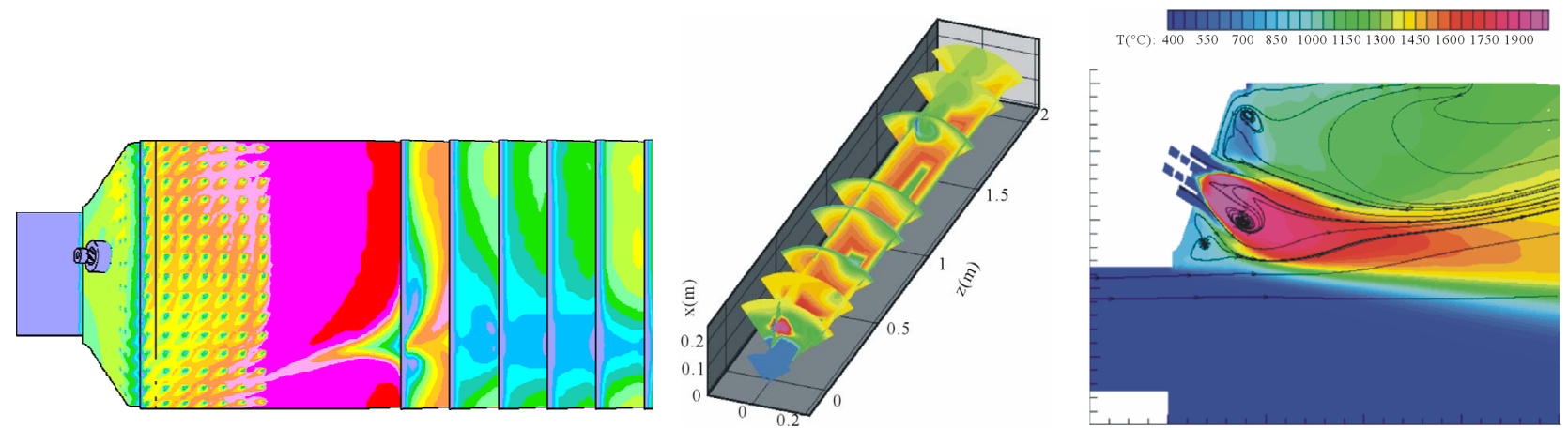

Figure 6. Left: combustion chamber surface temperature. Centre: global flow temperature in the combustion chamber, trace effects of the pilot burner, the cooling inlet rows and the dilution hole can be appreciated. Right: flow temperature and streamlines on the plane containing the chamber and the pilot burner axes.

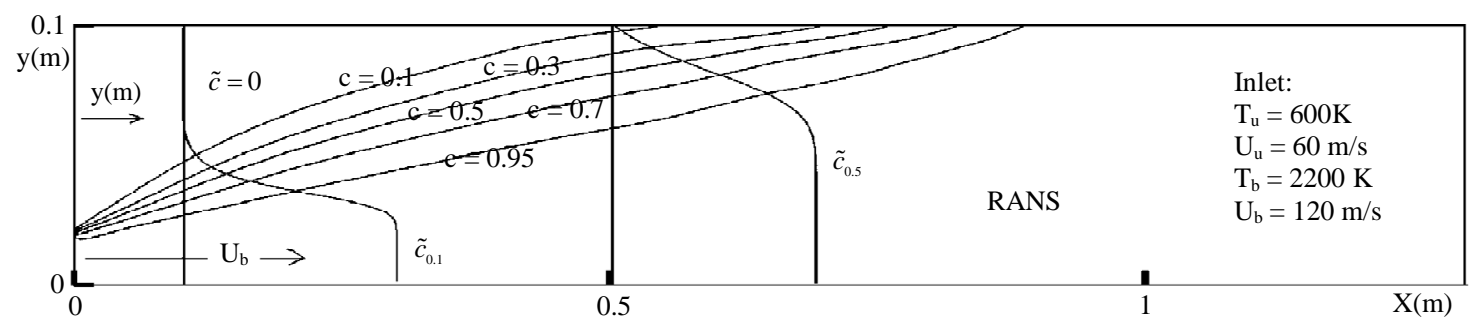

(a)

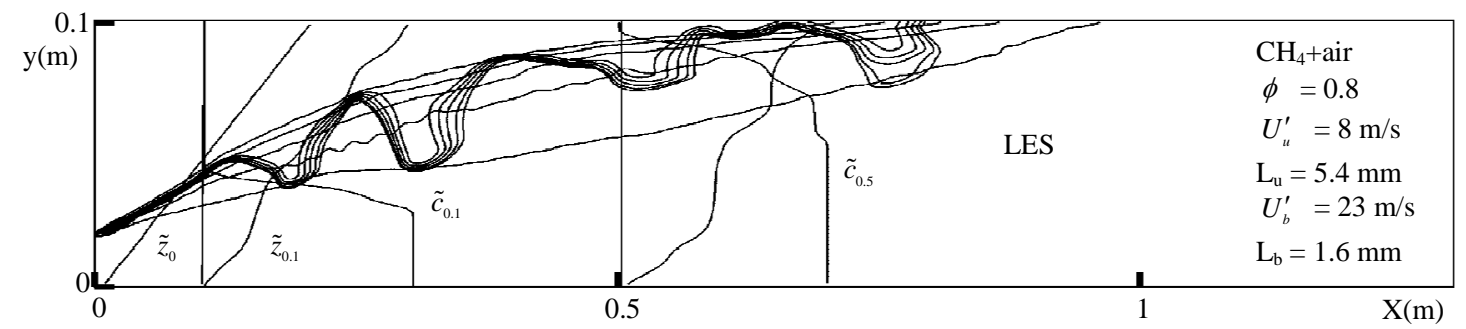

(b)

Figure 7. Average and instantaneous field of progress variable (a): RANS simulation, (b) corresponding LES. 

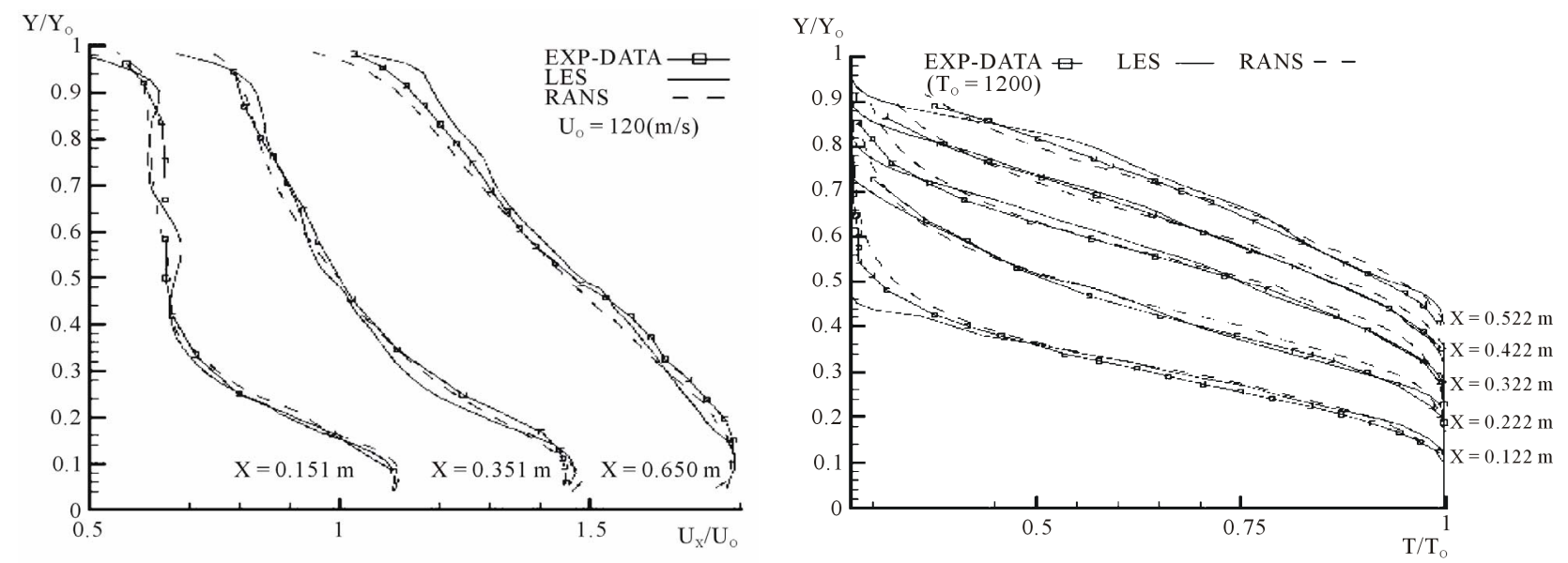

Figure 8. Comparison of axial velocity (left) and temperature (right) with experimental data.

tration is controlled only by turbulent diffusion, while the flux of the progress variable is controlled by turbulence and the gasdynamic mechanism: different pressure-driven acceleration in the flame of relatively heavy reactants and light products. The balance between turbulent and gasdynamic mechanisms controls observed in the premixed flame transition from gradient to counter-gradient flux of the reacting $c$. It is to remark that LES describe this transition without any additional model [6]. In the RANS version of the TFC model we overcame this problem by including of the gasdynamic contribution in the model chemical source [2]. So the transport term in Equation (4) is controlled only by gradient turbulent diffusion and hence there is no need to model in practical simulation the counter-gradient transport phenomenon.

\subsection{Simplified Version of the GE10 Combustor}

To test the Joint RANS/LES approach, we performed LES of a simplified geometry of the first version of the GE burner previously described (the cooling jets and the pilot system were not meshed). We used Fluent code and simulated a $60^{\circ}$ degree section of the burner applying periodic boundary condition at the lateral sections. LES average shows good agreement with RANS data. The non-stationary images of the flow field give an idea of the effects of large scale vortices on the flame displacements.

In Figure 9(a) RANS and instantaneous LES progress variable are overlapping on a section of the burner to show the convolution of the instantaneous reacting zone, which travels and is stretched by vortex shedding from the geometrical step. Figure 9(b) clearly shows the turbulent structure originated at the inlet step and how they evolve along the flow. LES gives the possibility to understand the trend of variation of the flame shape changing operative conditions and boundary conditions. It is possible to create 3D visualizations to see the effects of the vortex structures in the circumferential direction as shown in Figure 10. Our joint RANS/LES approach, obviously, does not exclude the possibility to use LES modeling, that is independent on the RANS one and based on using common now Smagorinsky model of the sub-grid viscosity. In this connection, we would like to mention the paper [11], which is devoted to the comparison of predictions of the premixed flame anchoring in the double cone gas turbine burner using independent RANS and LES approaches based on our TFC combustion model. We notice only that our joint RANS/LES approach permits to reduce significantly computation time of LES.

\section{Conclusions}

The presented results consist of two parts:

1) Comprehensive (wherever possible) RANS numerical simulations of aerodynamic systems of two versions of the GE10 gas turbine combustor (with nonpremixed and swirled premixed pilot flames): cold mixing chambers of the combustor and the premixed pilot burner, aerodynamic of the main and pilot flames together with an actual system of air cooling jets.

2) Numerical illustrations of our original joint RANS/ LES approach applied to the gas turbine combustion (using the standard model situation and a simplified version of the GE10 combustor), which can be an effective and economical tool for the analysis of both stationary mean and nonstationary fields of parameters.

All simulations where performed in the context of the TFC combustion model, which, in particular yielded reasonable agreement between RANS and LES subproblems.

Our general conclusion is that RANS simulations re- 


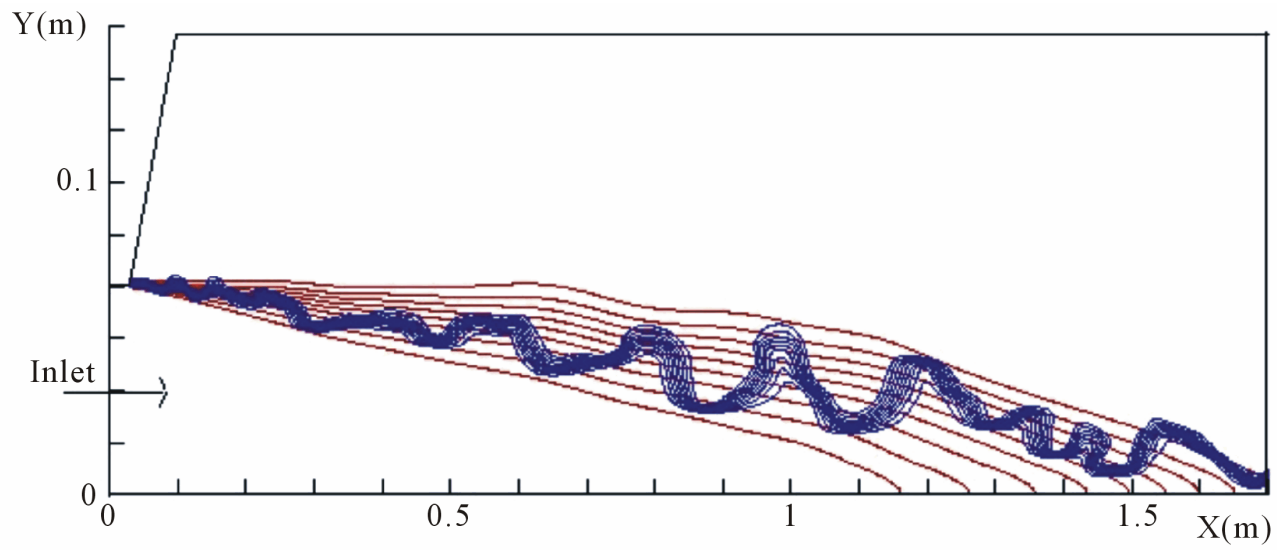

(a)

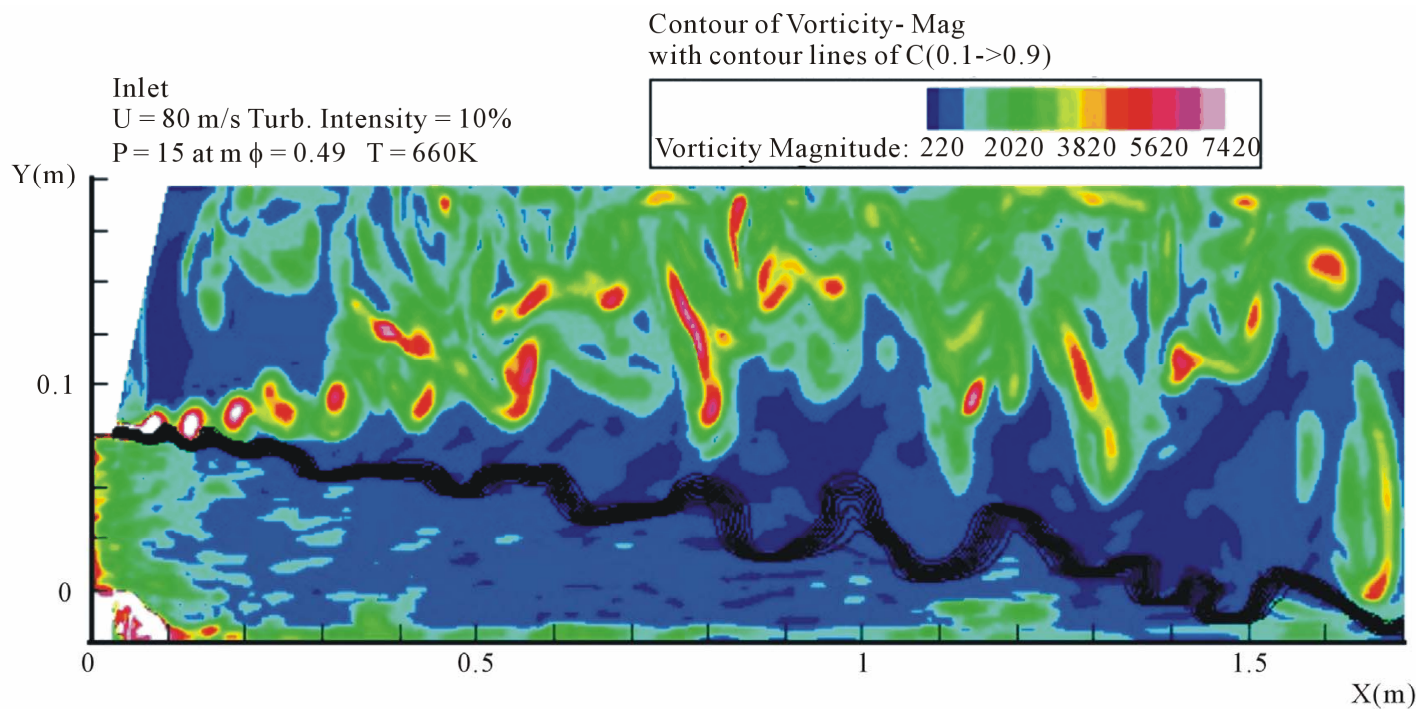

(b)

Figure 9. 2D visualization of middle-section. (a): Contour lines of progress variable (from 0.1 to 0.9 step 0.2 ) for RANS (brown) and instantaneous LES (blue); (b); (b) iso-surfaces of LES and the field of the vorticity.
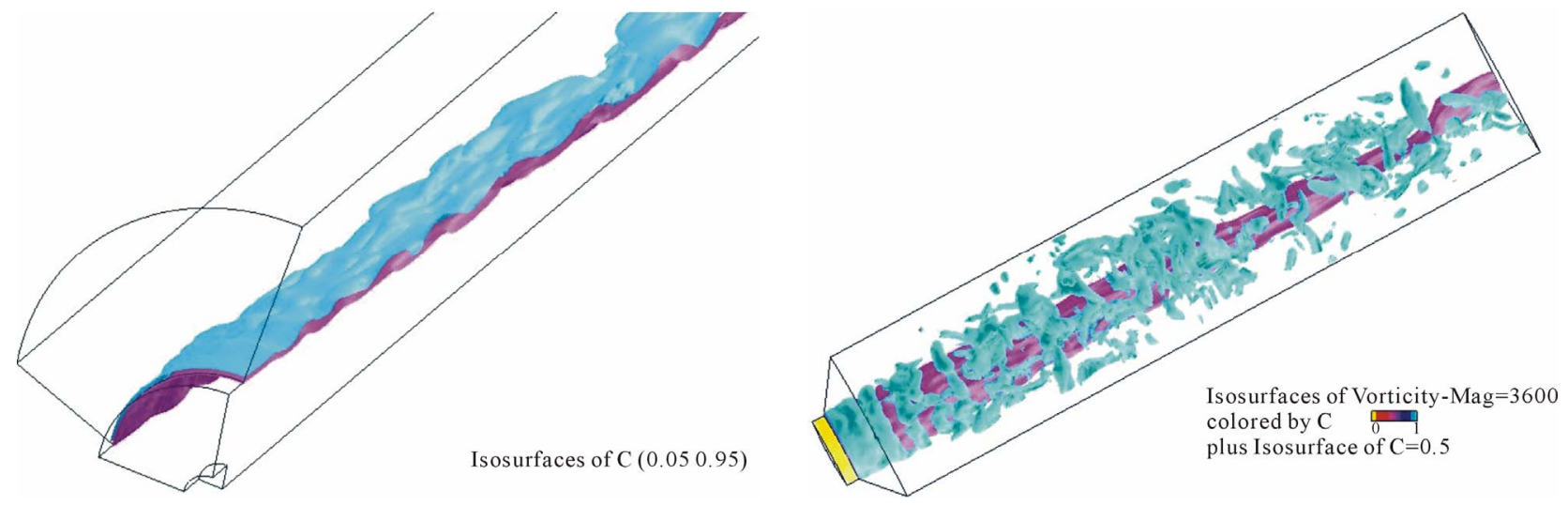

Figure 10. 3D visualization. Two isosurfaces of the progress variable (left) and the field of the vorticity (right).

main a necessary (but not sufficient) tool for practical numerical analysis of the gas turbine premixed combus- tion and the joint RANS/LES approach can be a useful tool for investigation of non-stationary characteristics of 
the flow, including unsteady combustion regimes. It seems that complete replacement of the RANS tool by the LES one is untimely at least for gas turbine applications.

\section{References}

[1] V. L. Zimont, "A Theory of Turbulent Premixed Combustion at High Reynolds Numbers," Combustion, Explosion, and Shock Waves, Vol. 15, No. 3, 1979, pp. 305-311. doi:10.1007/BF00785062

[2] V. L. Zimont, "Gas Premixed Combustion at High Turbulence. Turbulent Flame Closure Combustion Model,” Experimental Thermal and Fluent Science, Vol. 21, No. 1-3, 2000, pp. 179-186. doi:10.1016/S0894-1777(99)00069-2

[3] V. Zimont, "Kolmogorov's Legacy and Turbulent PreMixed Combustion Modelling," In: W. J. Carey, Ed., New Developments in Combustion Research, Nova Science Publishers, New York, 2006, pp. 1-93.

[4] V. Zimont, W. Polifke, M. Bettelini and W. Weisenstein, "An Efficient Computational Model for Premixed Turbulent Combustion at High Reynolds Numbers Based on a Turbulent Flame Speed Closure,” Journal of Engineering for Gas Turbines and Power (Transactions of the ASME), Vol. 120, No. 3, 1998, pp. 526-532. doi:10.1115/1.2818178

[5] V. Biagioli, L. Zimont and K. J. Syed "Modelling and Nu- merical Simulation of Turbulent Combustion in DLE Burners Based on a Turbulent Flame Speed Approach,” International Joint Power Generation Conference, New Orleans, 4-7 June 2001, pp. 1-14.

[6] V. L. Zimont and V. Battaglia, "Joint RANS/LES Approach to Premixed Flame Modelling in the Context of the TFC Combustion Model, Flow, Turbulence and Combustion”, Vol. 77, No. 1-4, 2006, pp. 305-331.

[7] T. Poinsot and L. Selle, "LES and Acoustic Analysis of Combustion Instabilities in Gas Turbine," Plenary Lectures ECCOMAS-Computational Combustion Symposium, Lisbon, 21-24 June 2005.

[8] V. L. Zimont and A. N. Lipatnikov, "A Numerical Model of Premixed Turbulent Combustion of Gases," Chemical Physics Reports, Vol. 14, No. 7, 1995, pp. 993-1025.

[9] V. P. Karpov, A. N. Lipatnikov and V. L. Zimont, "Test of the Engineering Premixed Combustion Model," Symposium (International) on Combustion, Vol. 26, No. 1, 1996, pp. 249-261.

[10] P. Moreau, "Turbulent Flame Development in a High Velocity Premixed Flow,” American Institute of Aeronautics and Astronautics, Aerospace Sciences Meeting, Los Angeles, 24-26 January 1977.

[11] F. Biagioli, "Stabilization Mechanism of Turbulent PreMixed Flames in Strongly Swirled Flows," Combustion Theory and Modelling, Vol. 10, No. 3, 2006, pp. 389-412. doi:10.1080/13647830500448347 